\title{
Texture Classification Of Sea Turtle Shell BASEd ON COLOR FEATURES: COLOR Histograms AND CHROMATICITY MOMENTS
}

\author{
Wdnei R. da Paixão ${ }^{1}$, Thiago M. Paixão ${ }^{2}$, Mateus C. B. da Costa ${ }^{3}$, Jefferson O. \\ Andrade $^{4}$, Flavio G. Pereira ${ }^{5}$, Karin S. Komati ${ }^{6}$ \\ ${ }^{1,5,6}$ Postgraduate Program in Control and Automation Engineering ${ }^{2,3,4}$ Department of \\ Informatics Instituto Federal do Esp’́1rito Santo (IFES), Serra, ES, Brazil.
}

\begin{abstract}
A collaborative system for cataloging sea turtles activity that supports picture/video content demands automated solutions for data classification and analysis. This work assumes that the color characteristics of the carapace are su $\square$ cient to classify each species of sea turtles, unlikely to the traditional method that classifies sea turtles manually based on the counting of their shell scales, and the shape of their head. Particularly, the aim of this study is to compare two features extraction techniques based on color, Color Histograms and Chromaticity Moments, combined with two classification methods, K-nearest neighbors $(K N N)$ and Support Vector Machine (SVM), identifying which combination of techniques has a higher $e \square$ ectiveness rate for classifying the five species of sea turtles found along the Brazilian coast. The results showed that the combination using Chromaticity Moments with the KNN classifier presented quantitatively better results for most species of turtles with global accuracy value of 0.74 and accuracy of $100 \%$ for the Leatherback sea turtle, while the descriptor of Color Histograms proved to be less precise, independent of the classifier. This work demonstrate that is possible to use a statistical approach to assist the job of a specialist when identifying species of sea turtle.
\end{abstract}

\section{KEYWORDS}

Sea turtle shell, Color texture classification, Color histograms, Chromaticity moments, KNN, Support vector machine.

\section{INTRODUCTION}

Among seven species of sea turtles found worldwide, five are encountered in Brazil, and all of them are in the red list of threatened species according to the IUCN (International Union for Conservation of Nature) [1]. Sea turtles have a long and complex life cicle, whereas the adulthood of these animals starts from 25 years old, leading to complication on the specie development [2], and it may get worse because of the e $\square$ ects of climate change [3]. However, the extinction of sea turtles has been combated by environmental organizations such as the TamarICMBio [4].

In Brazil, information about sea turtles began to be collected in the early 80 's by identifying the coastal areas of reproduction [5]. As a natural consequence of research activity over the years, such areas have experienced the emergence of research centers, as well as the development of environmental education projects focused on sea turtle conservation. The prominent example of

DOI : 10.5121/ijaia.2018.9205 
this is the [4], a federal program of research applied to marine wildlife preservation. Since 1982, the project have devoted e $\square$ orts to collect sea turtle data by tagging females, analyzing the spawning phases and mapping the life-cycle of these animals using geolocation information. To manage this data the SITAMAR system was developed [6]. The SITAMAR is a restricted-access information system which has remarkably improved storing, querying, and analysis of gathered data.

Despite the progress achieved with SITAMAR, past experiences showed that coastal community participation is essential to assist biologists by providing them information about the turtles daily routine, which has motivated TAMAR-ICMBIO leaders to make the system open-access in the near future [7]. This collaborative aspect of the system, however, implies the continued and fast growth of the database, which in conjunction with the variety of turtle species and the limited number of taxonomists or experts on species classification, leads to a big challenge for future studies, as [8] points out.

Current collaborative systems are intrinsically related to mobile technologies that enable, among other things, the users to share text, geolocation, activities, and also image content. SITAMAR is expected to provide a mobile interface that supports on demand uploading of picture/video content of turtle activity, which demands automated solutions for data organization and analysis of this ever-increasing database.This work, particularly, focuses on the problem of turtle species classification based on computer vision techniques. For instance, there are a great $\mathrm{e} \square$ ort on studies for automating the classification of animals by using machine learning methods.

Usually, biology experts classify turtles manually based on the counting of their shell scales, and the shape of their head [10]. Nonetheless, [8] and [11] showed that computer vision techniques are suitable to classify animal species with distinct patterns of color and texture in their corporeal formation. Therefore, scientists in the area of protection of marine turtles have been trying to extract unique features for identifying to which species an individual animal belongs [12, 13, 14]. Also present keys to identify sea turtles from photographs which goes towards a non-invasive method for identification $[15,12,16]$. However, [14] shows that color texture features outperform the shape feature for the recognition of sea turtles species. In this work, we investigated two color-based descriptors for automated species classification: a) color histogram features [17]; and b) chromaticity moments [18]. Although it di $\square$ ers from the human-based classification criteria, these features were adopted based on the distinctive shell color patterns observed for sea turtle species, as depicted in Fig. 1.

The samples of the five sea turtle species found along the Brazilian coast in their natural environment [19] is presented in Figure 1. It is also showed, beside each turtle picture, a rectangular patch cropped from the shell region of the respective turtle, as well as the RGB histogram related to such patch. The histogram for the green turtle, for example, spreads over a wide color range for the three channels (RGB), while the Leatherback turtle histogram tends to concentrate at low values (dark colors).The Loggerhead turtle (or Caretta Caretta) is reddish, in which the peaks of the green and blue channels are very close and have values smaller than the peak of the red channel, which becomes more central in the histogram; The Hawksbill Turtle (or Eretmochelys Imbricata) also has dark colors, but not as much as the Leatherback. While the Olive Ridley turtle (or Lepidochelys Olivacea) has more centralized peaks on its channels. 
Different studies argue that supervised machine learning techniques such as Artificial Neural Networks (ANN) [14, 20, 21],Naive Bayes [22, 23], Linear Discriminant Analysis (LDA) [24], Decision Trees [25], KNN [23] and Support Vector Machines (SVM) [23]. All these cited works have shown that automated species identification have higher accuracy when compared to human classification.
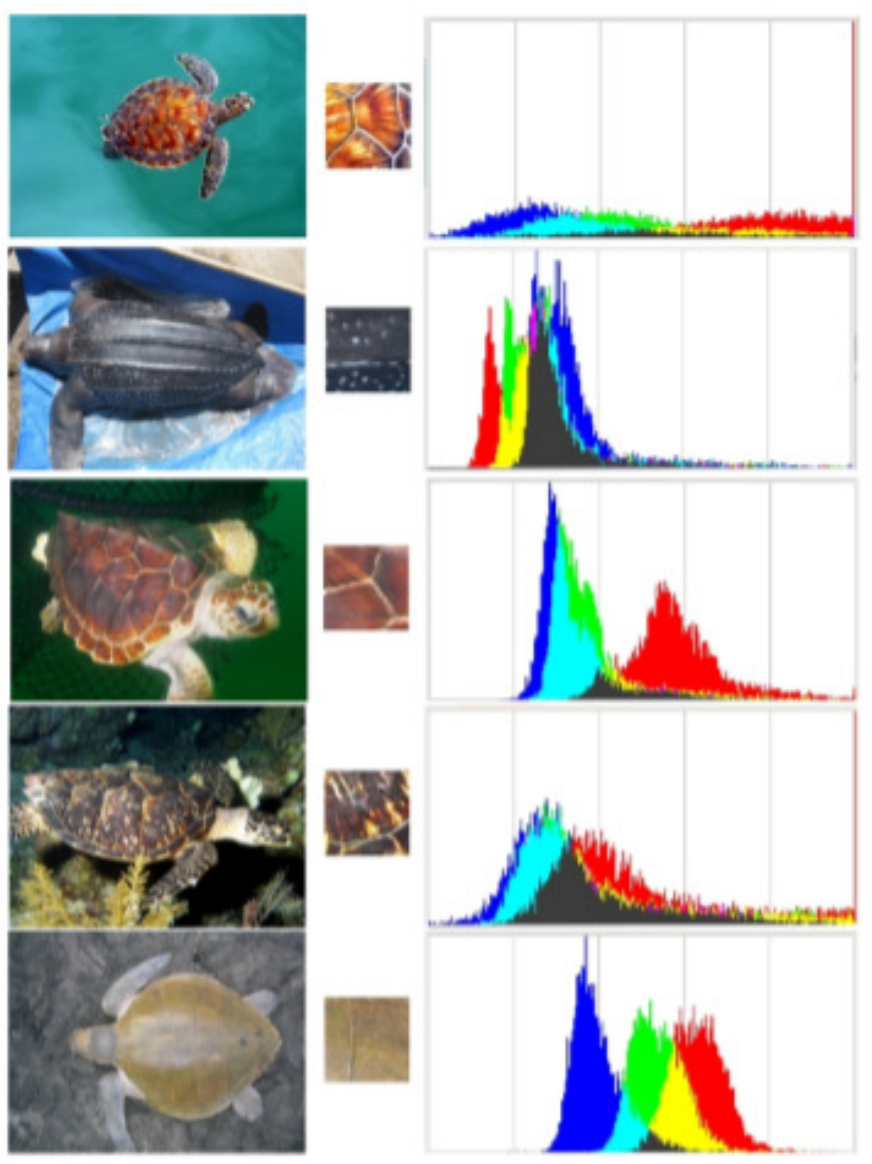

Figure 1: Sea turtle species found along Brazilian coast: (a) Green turtle (Chelonia mydas); (b) Leatherback turtle (Dermochelys coriacea); (c) Loggerhead turtle (Caretta caretta); (d) Hawksbill turtle (Eretmochelys

imbricata), (e) Olive ridley turtle (Lepidochelys olivacea). The middle column exhibits texture shell samples (rectangular patches) for each turtle depicted in the first column, as the rightmost column shows the RGB histograms computed for each texture sample, adapted from [9].

The main contribution of this study is the evaluation of the previously mentioned color descriptors in conjunction to K-Nearest Neighbor (KNN) [26] and Support Vector Machine (SVM) [27] techniques for sea turtle species classification. The image dataset, which is comprised solely of patches of turtle shell, was assembled specifically for the experiments conducted here, thus can be viewed as another contribution of our research1.

\footnotetext{
${ }^{1}$ https://github.com/wdnei/identificarTartarugas/tree/master/v3/samples
} 


\section{Materials AND Methods}

\subsection{Data collection}

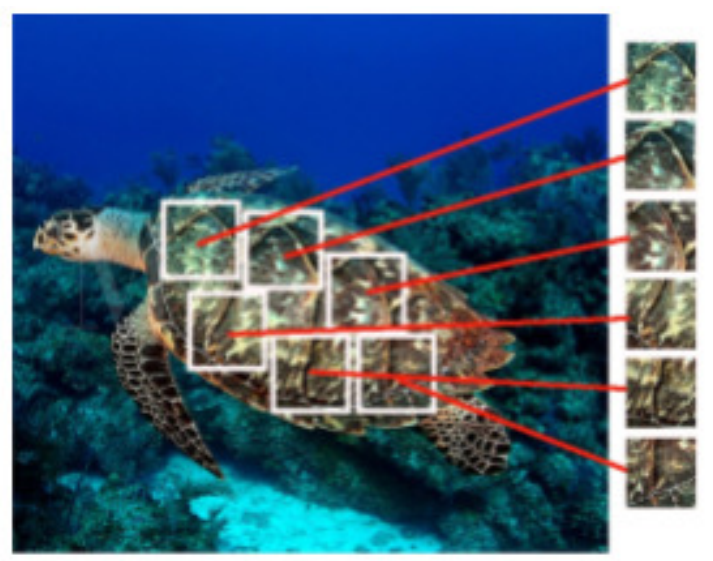

Figure 2: Six $80 \times 80$ texture samples extracted from an exemplar of turtle (adapted from [28]).

The image dataset used in the experiments was assembled specifically for this study. First, we collected 15 photos of turtles (three per specie) from public online repositories, being the photos RGB images with average resolution of $800 \times 600$ pixels. For each image, six square $80 \times 80$ texture patches were manually cropped from the region that encloses the turtle shell (Figure 2), therefore totalling 90 texture patches (18 per sea turtle specie).

\subsection{Color features}

The classification task addressed in this work relies fully on the color information present in sea turtle shells. Such information must be encoded as a n-dimensional feature vector $\mathrm{x}=$ $\left(\mathrm{x}_{1}, \mathrm{x}_{2}, \ldots, \mathrm{x}_{\mathrm{n}}\right)^{\mathrm{T}}$, i.e., a real-valued vector representation suitable for classification tools. The ensuing sections presents the theoretical background of color features extraction by means of color histogram features and chromaticity moments, the two colors descriptors explored in this study.

\subsubsection{Color histogram features}

The first analyzed feature extractor consists of five statistics extracted from color distributions. Let $\mathrm{h}$ be a histogram for a single color channel of a $\mathrm{M} \times \mathrm{N}$ image. The probability mass function can be obtained by normalizing $\mathrm{h}$ by the total number of pixels from the source image. In mathematical notation, we have

$$
p(l)=\frac{h(l)}{N M}, l=0,1, \ldots, L-1,
$$


where 1 denotes an intensity level, and L denotes the number of possible intensity levels (here we adopted $\mathrm{L}=255$ ). The statistical measures used as features can be computed straight from the probability mass function as follow:

$$
\begin{aligned}
& \text { - Mean: } \mu=\sum_{l=0}^{L-1} l p(l) \\
& \text { - Variance: } \sigma^{2}=\sum_{l=0}^{L-1}(l-\mu)^{2} p(l) \\
& \text { - Kurtosis: } \mu_{4}=\sigma^{-4} \sum_{l=0}^{L-1}(l-\mu)^{4} p(l)-3 \\
& \text { - Energy: } E=\sum_{l=0}^{L-1} p(l)^{2} \\
& \text { - Entropy: } H=-1 \sum_{l=\mathrm{n}}^{L-1} p(l) \log _{2} p(l)
\end{aligned}
$$

The mean, the more intuitive measure, is the average intensity level of the image, while the variance quantifies how much the color distribution spreads in relation to the mean. Kurtosis is also a measure of sparsity and quantifies how flat (or peaky) is the histogram. Energy evaluates the textural uniformity of the image, where higher energy values associated to images with few colors. Finally, we use the entropy to measure the disorder degree of image data [17].In this study, statistical measures are independently computed for the RGB channels, therefore each measure gives rise to three elements in the feature vector, totaling 15 features as described in the expression: $\left(\mu_{\mathrm{r}}, \mu_{\mathrm{g}}, \mu_{\mathrm{b}}, \sigma_{\mathrm{r}}^{2}, \sigma_{\mathrm{g}}^{2}, \sigma_{\mathrm{b}}^{2}, \mu_{\mathrm{r}}^{4}, \mu_{\mathrm{g}}^{4}, \mu_{\mathrm{b}}^{4}, \mathrm{E}_{\mathrm{r}}, \mathrm{E}_{\mathrm{g}}, \mathrm{E}_{\mathrm{b}}, \mathrm{H}_{\mathrm{r}}, \mathrm{H}_{\mathrm{g}}, \mathrm{H}_{\mathrm{b}}\right)$.

\subsubsection{Chromaticity moments}

[18] introduced the chromaticity moments as an e $\square$ cient descriptor for color texture classification. The chromaticity information of an image is described by the occurrence of certain colors in the CIE-XYZ space (chromaticity diagram), as well as by the number of occurrences (pixels) of each color (chromaticity distribution). [18] makes use of mathematical moments to summarize both the chromaticity diagram and distribution, which characterizes the concept of chromaticity moments. For a more formal definition, consider an $\mathrm{M} \times \mathrm{N}$ image $\mathrm{I}$. The chromaticity diagram for I is defined as follows:

$$
T(x, y)= \begin{cases}1, & \text { if } \exists i, j: I(i, j) \text { produces }(x, y), \\ 0, & \text { otherwise, }\end{cases}
$$

The respective chromaticity distribution is a $2 \mathrm{D}$ histogram given by:

$$
D(x, y)=\mid\{(i, j): I(i, j) \text { produces }(x, y)\} \mid
$$

The Figure 3 shows how $\mathrm{T}$ and $\mathrm{D}$ are distributed. $\mathrm{T}(\mathrm{x}, \mathrm{y})$ and $\mathrm{D}(\mathrm{x}, \mathrm{y})$ can be viewed as functions over which the respective moments of order $(m+1)$ can be calculated: 


$$
M_{P_{m, d},}(x, y)=\sum_{x=0}^{X_{y}-1} \sum_{y=0}^{Y_{y}-1} x^{m} y^{l} T(x, y)
$$
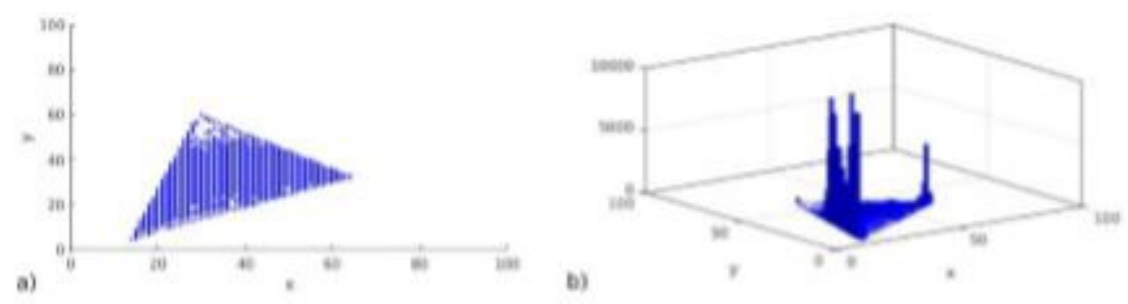

Figure 3: An example of $\mathrm{x}$; $\mathrm{y}$ chromaticities: (a) the two-dimensional trace (T), (b) the two-dimensional distribution (D).([18]).

where $X_{S}=Y_{S}=100$, following the normalization adopted by [18]. A set of moments can be obtained by assigning di $\square$ erent values for $(\mathrm{m}, \mathrm{l})$, being that the insertion/removal of a new moment can $a \square$ ect the classification performance. This work adopts the five combinations in $(0,0),(1,0),(2,0),(0,1),(0,2)$ for each moment definition, which leads to the following feature vector: $\left(\mathrm{M}_{\mathrm{T}}{ }^{0,0}, \mathrm{M}_{\mathrm{T}}{ }^{1,0}, \mathrm{M}_{\mathrm{T}}{ }^{0,1}, \mathrm{M}_{\mathrm{T}}{ }^{2,0}, \mathrm{M}_{\mathrm{T}}{ }^{0,2}, \mathrm{M}_{\mathrm{D}}{ }^{0,0}, \mathrm{M}_{\mathrm{D}}{ }^{1,0}, \mathrm{M}_{\mathrm{D}}{ }^{0,1}, \mathrm{M}_{\mathrm{D}}{ }^{2,0}, \mathrm{M}_{\mathrm{D}}{ }^{0,2}\right)$.

\subsection{Classification}

This work was based on a supervised classification approach. Supervised classification aims to assign a label to an unknown object given a training dataset consisting of known (or labeled) objects. Such objects are represent by a proper set of features embedded in a multi-dimensional space, the (feature space). In this space, a proper choice of features makes distinct-class objects to occupy regions as disjoint as possible, while same-class objects tend to be restricted to compact regions. This fact is explored in di $\square$ erent machine learning algorithms such as Support vector machines (SVM) [27] and K-nearest neighbors (KNN) [26], the two classification techniques investigated in this work. The general workflow described above is show on Figure 4.

In a broader view, SVMs set linear decision boundaries (hyper-planes) as far as possible from example points [27], which comprehend the features extracted from the training dataset. For nonlinear classification, the kernel trick can be employed, so that the training data is transposed into a higher-dimensional feature space where data can be easily linearly separated. KNN, in turn, is a simple non-linear classification technique based on a voting scheme. Basically, a new object is labeled with the majority vote of its $\mathrm{K}$ nearest neighbors, that is, a subset of the training objects (example points) with the $\mathrm{K}$ most similar elements given a distance metric in the feature space. 


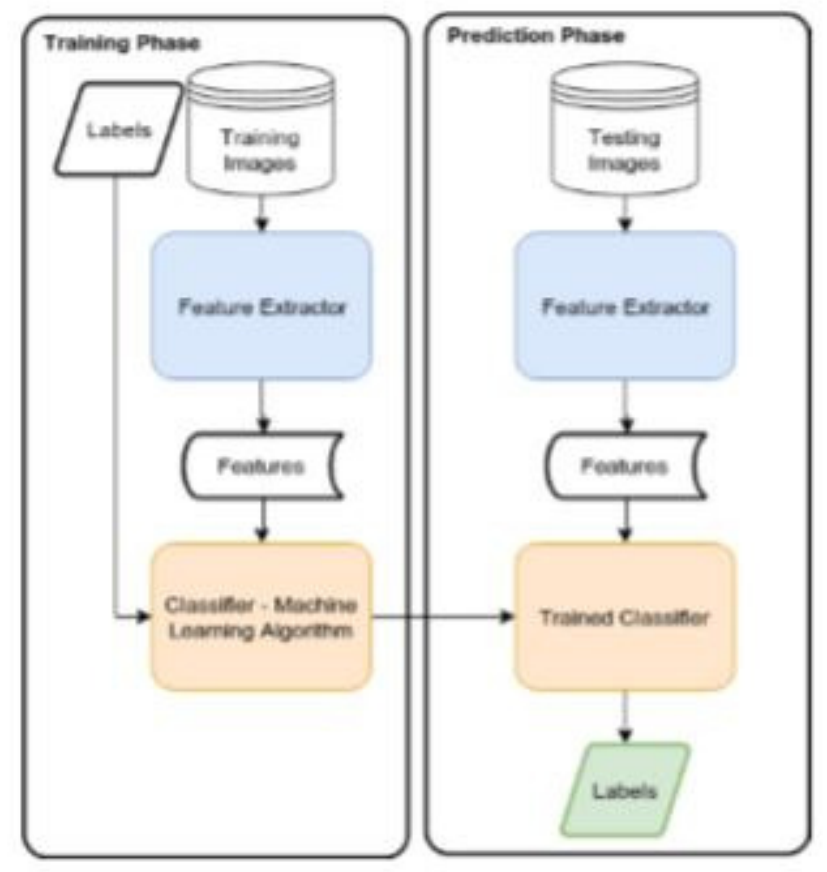

Figure 4: Supervised classification process used in this study.

\section{EXPERIMENTAl RESUltS AND DisCUSSION}

All experiments were carried out in Python using the Open CV library version 2.4 [29]. The color histogram features and the chromaticity moments were tested against the KNN and SVM classifiers. For KNN, it was set $K=3$ and the Euclidean distance was used as distance metric. As for SVM, the linear kernel was employed. Performance statistics were estimated with crossvalidation techniques Leave-one-out and $k$-fold [30] $(k=10)$ were used to assess the performance of each descriptor-classifier combination, preserving class proportions.

The results of those experiments were summarized in confusion matrices. From those matrices three performance measures were calculated: precision, recall and f-measure. Fmeasure is expressed by the harmonic mean of precision and recall, where the best values are close to 1 [31]. The obtained values for these measures are illustrated by means of network and bars graphs. Also, the class and feature extractor names were shortened to facilitate the classification analyses, as shown in Table 1.

Table 2 and Table 3 show results of precision and recall for each sea turtle species. It is easy to perceive that Lepido is the class that presents more problems and variation on precision results, being the worst case 0.23 , which shows a di $\square$ culty to represent this class on terms of texture. In contrast, Dermo and Eretmo show good results that could reach 0.95 and 0.91 on precision values, and 1 and 0.89 on recall values.

Figure 5a shows a big di $\square$ erence when using distinct classifiers. It is easy to notice that 
International Journal of Artificial Intelligence and Applications (IJAIA), Vol.9, No.2, March 2018

Table 1: Abbreviations used on the classification analyses

\begin{tabular}{|l|l|}
\hline Abbreviation & Definition \\
\hline Caretta & Caretta caretta \\
\hline Chelonia & Chelonia mydas \\
\hline Dermo & Dermochelys coriacea \\
\hline Eretmo & Eretmochelys imbricata \\
\hline Lepido & Lepidochelys olivacea \\
\hline HCo & Color Histogram \\
\hline MoCr & Chromaticity Moments \\
\hline
\end{tabular}

Table 2: Precision and Recall on each combination of feature descriptor and classifier on the approach $K$ Fold.

\begin{tabular}{|l|l|l|l|l|l|l|}
\hline \multicolumn{7}{|c|}{ HiCo =>KNN; k-fold-10 } \\
\hline & Caretta & Chelonia & Dermo & Eretmo & Lepido \\
\hline Precision & 0.65 & 0.79 & 0.37 & 0.84 & 0.39 \\
\hline Recall & 0.72 & 0.61 & 0.39 & 0.89 & 0.39 \\
\hline \multicolumn{7}{|c|}{ HiCo =>SVM; k-fold-10 } \\
\hline & Caretta & Chelonia & Dermo & Eretmo & Lepido \\
\hline Precision & 0.35 & 0.50 & 0.62 & 0.89 & 0.36 \\
\hline Recall & 0.65 & 0.50 & 0.72 & 0.44 & 0.22 \\
\hline \multicolumn{7}{|c|}{ MoCr =>KNN; k-fold-10 } \\
\hline Precision & 0.56 & 0.79 & 0.95 & 0.91 & 0.58 \\
\hline Recall & 0.83 & 0.61 & 1,00 & 0.56 & 0.61 \\
\hline \multicolumn{7}{|c|}{ MoCr =>SVM; k-fold-10 } \\
\hline Precision & 0.68 & 0.35 & 0.90 & 0.50 & 0.62 \\
\hline Recall & 0.72 & 0.44 & 1,00 & 0.56 & 0.28 \\
\hline
\end{tabular}

Table 3: Precision and Recall on each combination of feature descriptor and classifier on the approach Leave-One-Out.

\begin{tabular}{|c|c|c|c|c|c|}
\hline \multicolumn{6}{|c|}{ HiCo $=>\mathrm{KNN}$; Leave-One-Out } \\
\hline & Caretta & Chelonia & Dermo & Bretmo & Lepido \\
\hline Precision & 0.60 & 0.73 & 0.47 & 0.80 & 0.44 \\
\hline Recull & 0.67 & 0.61 & 0.50 & 0.89 & 0.39 \\
\hline \multicolumn{6}{|c|}{ HiCo =>SVM; Leave-One-Out } \\
\hline & Caretta & Chelonia & Dermo & Eretmo & Lepido \\
\hline Precision & 0.36 & 0.64 & 0.60 & 0.77 & 0.23 \\
\hline Recall & 0.67 & 0.39 & 0.67 & 0.56 & 0.17 \\
\hline \multicolumn{6}{|c|}{ MoCr $=>$ KNN; Leave-One-Out } \\
\hline & Caretta & Chelonia & Dermo & Eretmo & Lepido \\
\hline Precision & 0.62 & 0.79 & 0.95 & 0.85 & 0.60 \\
\hline Recall & 0.83 & 0.61 & 1,00 & 0.61 & 0.67 \\
\hline \multicolumn{6}{|c|}{ MoCr $=>$ SVM; Leave-Ono-Out } \\
\hline & Caretta & Chelonia & Dermo & Eretmo & Lepido \\
\hline Precision & 0.68 & 0.17 & 0.95 & 0.47 & 0.50 \\
\hline Recall & 0.72 & 0.33 & 1,00 & 0.39 & 0.06 \\
\hline
\end{tabular}

the best performance was reached by the KNN classifier, since almost all values are closer to the edge of the graph. Therefore, in class Dermo the classification achieved a very 


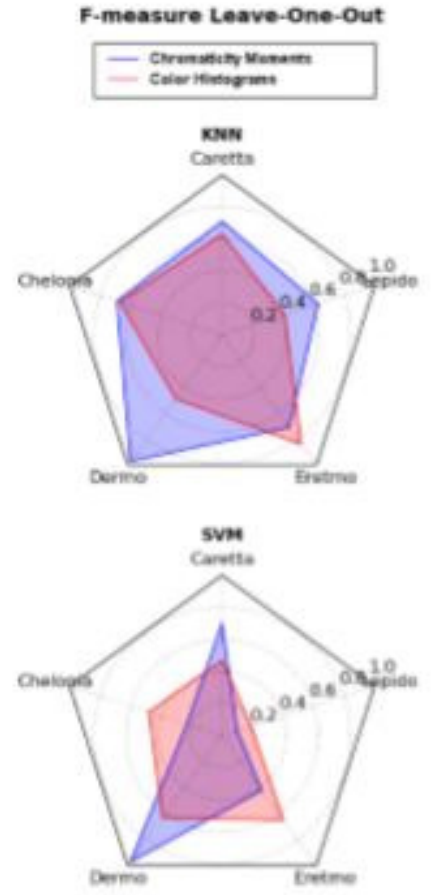

(a) Leave-One-Out

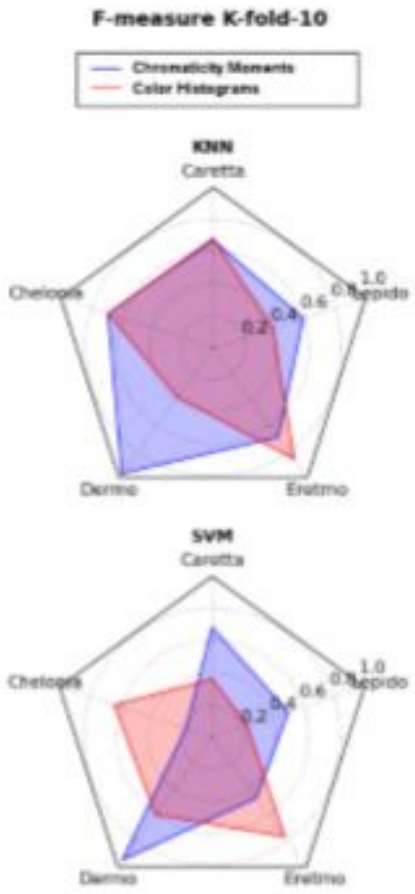

(b) $K$-fold $=10$

Figure 5: F-measure analysis

high value, when using the method of Chromaticity Moments, regardless of the classifier. However, when applying Color Histograms in the same class, the values were below to 0.6, showing a great di $\square$ erence in relation to the feature descriptors. Likewise, the class Caretta also had the same behavior, and had the best result, 0.7 when using Chromaticity Moments on both classifiers, and having the worst case for combination Color Histograms and SVM, reaching 0.47. In contrast, the behavior of other classes was considerably di $\square$ erent when changing the combination of the descriptor and classifier. The class Chelonia showed a good performance on the methods Chromaticity Moments and KNN, but had the worst performance when changing the classifier, going from 0.69 to 0.22 , showing that the choice of classifier is important for the methods proposed in this work.

Another divergence was verified for the class Lepido. The class that proved to be the most di $\square$ culty to classify. This class had its best performance when combining Chromaticity Moments and KNN, but when combining the same descriptor method with the SVM classifier, there was a drastic fall in the value of F-measure reaching 0.1. This class causes the SVM classifier to behave very poorly, generating erroneous predictions.

The class Eretmo presented a behavior totally di $\square$ erent from the others, since its best case 
was achieved by using the method of Color Histograms, even when changing the classifier. This shows that the textures of this class present information that are best captured by the Color Histograms method.

Finally, the Figure $5 \mathrm{~b}$ shows the $k$-fold cross-validation method. It is important to note that, for the SVM classifier, it revealed big changes in relation to the Leave-One-Out analysis. The class Lepido presented the most improvement, especially when using the Chromaticity Moment descriptor. Also, as already predicted, the graph of the KNN classifier shows that there was a significant decay in the values, since not all the training images of each class were allocated as in the Leave-One-Out analysis. Maybe, the great variation for the SVM chart is due to the fact of the classifier was less confused by having fewer images of the class Lepido, leading to a small increase in the values of $F$-measure.

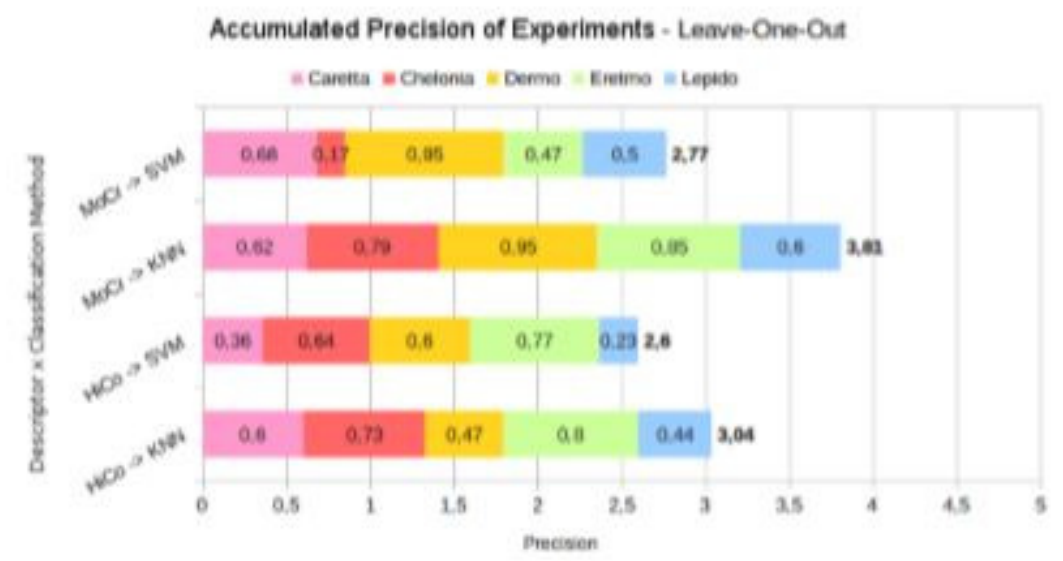

Figure 6: Accumulated Precision for Leave-One-Out

Figure 6 presents the cumulative precision sum of all tests performed for the Leave-OneOut approach, in the range of 0 to 5 , since it is the sum all five classes.

The best value found was 3.81 for the combination Chromaticity Moments and KNN as a classifier. Also, this combination was the only one for which the precision values of all classes were above 0.6 or $60 \%$. That is, the precision rate for this combination shows a higher confidence because the maximum error rate is $40 \%$. Only in the Caretta species this combination did not reach the best result, losing to the combination of Chromaticity Moments and the SVM classifier.

\section{Conclusion}

The Color Histograms descriptor combined with SVM presented more problems due to the characteristics of the images being represented in a more basic form; and also due to the fact that the classifier is not very precise in separating the presented characteristics and classes. The Chromaticity Moments method combined with the SVM classifier also displayed classification failures due to the lower accuracy of the classifier.

The results of Chromaticity Moments combined with the KNN classifier were clearly superior, probably due to a more precise class separation provided by the classifier. The results 
obtained were satisfying, especially for the species Dermochelys Coriacea, Eretmochelys Imbricata and Chelonia Mydas. Although, the classes Caretta Caretta and Lepidochelys Olivacea presented lower performance, it was still an improvement over the Color Histograms method.

The results presented in this paper demonstrate that it is possible to use the statistical approach in pattern recognition, but there are still room for improvement as the use of Deep Convolutional Neural Networks [32]. The database created for this work will be released for public use, as well as these results, making the community of researchers able to propose di $\square$ erent systems and techniques, and at the same time facilitate the comparison of the results. Hence, researchers and taxonomists can use the systems and save time in choosing which approach to use when developing an automatic classification system for sea turtle species.

\section{REFERENCES}

[1] The IUCN Red List of Threatened Species, "The iucn red list of threatened species," 2015. Last accessed 14 May 2015.

[2] Projeto TAMAR-ICMBIO, "Resultados," 2011. Last accessed 14 May 2015.

[3] G. Coro, C. Magliozzi, A. Ellenbroek, K. Kaschner, and P. Pagano, "Automatic classification of climate change e $\square$ ects on marine species distributions in 2050 using the AquaMaps model," Environmental and Ecological Statistics, vol. 23, pp. 155-180, dec 2015.

[4] Projeto TAMAR-ICMBIO, "Programa nacional de conserva ${ }_{s} \mathrm{c}^{\sim}$ ao de tartarugas marinhas," 2011. Last accessed 14 May 2015.

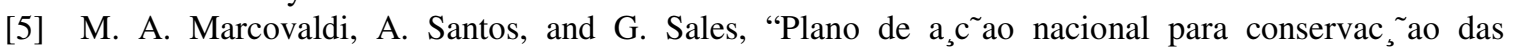
tartarugas marinhas.," Bras'́lia: ICMBio, 2011.

[6] Projeto TAMAR-ICMBIO, "Informac, oes gerais projeto tamar/icmbio," 2011. Last accessed 14 May 2015.

[7] MundoGEO, "Tamar usa gis e telemetria - mundogeo." http://mundogeo.com/blog/2004/ 03/14/tamarusa-gis-e-telemetria/, 2014. Last accessed 01 Nov 2015.

[8] A. Hern'andez-Serna and L. F. Jim'enez-Segura, "Automatic identification of species with neural networks," PeerJ, vol. 2, p. e563, 2014.

[9] Banco de Imagens Tamar, "Banco de imagens - projeto tamar," 2015. Last accessed 14 May 2015.

[10] R. M'arquez, "Fao species catalogue. sea turtles of the world: An annotated and illustrated catalogue of sea turtle species known to date.," FAO fisheries Synopsis, vol. 11, p. 81, 71990.

[11] C. Spampinato, D. Giordano, R. Di Salvo, Y.-H. J. Chen-Burger, R. B. Fisher, and G. Nadarajan, "Automatic fish classification for underwater species behavior understanding," in Proceedings of ACM international, (NY,USA), pp. 45-50, ACM, ACM, 2010.

[12] J. R. Lloyd, M. 'A. Maldonado, and R. Sta $\square$ ord, "Methods of developing user-friendly keys to identify green sea turtles (chelonia mydas 1.) from photographs," International Journal of Zoology, vol. 2012, 2012.

[13] G. Schofield, K. A. Katselidis, P. Dimopoulos, and J. D. Pantis, "Investigating the viability of photoidentification as an objective tool to study endangered sea turtle populations," Journal of Experimental Marine Biology and Ecology, vol. 360, no. 2, pp. 103-108, 2008.

[14] L. Pina, L. Rajamanickam, and S. Ng, "Feature extraction of the carapace for marine turtle species categorization," International Journal of Scientific Engineering and Technology, vol. 5, no. 9, pp. 425429, 2016.

[15] E. J. Pauwels, P. M. de Zeeuw, and D. M. Bounantony, "Leatherbacks matching by automated image recognition," in Industrial Conference on Data Mining, pp. 417-425, Springer, 2008.

[16] C. Jean, S. Ciccione, E. Talma, K. Ballorain, and J. Bourjea, "Photo-identification method for green and hawksbill turtles-first results from reunion," Indian ocean turtle NewsLeTTER, vol. 11, pp. 8-13, 2010. 
[17] S. Sergyan, "Color histogram features based image classification in content-based image retrieval systems," in Applied Machine Intelligence and Informatics, 2008. SAMI 2008. 6th International Symposium on, pp. 221-224, IEEE, 2008.

[18] G. Paschos, "Fast color texture recognition using chromaticity moments," Pattern Recogn. Lett., vol. 21, pp. 837-841, July 2000.

[19] M. A. Marcovaldi, V. Patiri, and J. Thom'e, "Projeto tamar-ibama: twenty-five years protecting brazilian sea turtles through a community-based conservation programme," Marit Stud, vol. 3, no. 2, pp. 39-62, 2005.

[20] S. J. Carter, I. P. Bell, J. J. Miller, and P. P. Gash, "Automated marine turtle photograph identification using artificial neural networks, with application to green turtles," Journal of Experimental Marine Biology and Ecology, vol. 452, pp. 105-110, 2014.

[21] P. Barr'e, B. C. St"over, K. F. Mu"ller, and V. Steinhage, "Leafnet: A computer vision system for automatic plant species identification," Ecological Informatics, 2017.

[22] R. Al-khurayji and A. Sameh, "An E $\square$ ective Arabic Text Classification Approach Based on Kernel Naive Bayes Classifier," International Journal of Artificial Intelligence Applications, pp. 01-10, nov 2017.

[23] F. S. Santana, A. H. R. Costa, F. S. Truzzi, F. L. Silva, S. L. Santos, T. M. Francoy, and A. M. Saraiva, "A reference process for automating bee species identification based on wing images and digital image processing," Ecological informatics, vol. 24, pp. 248-260, 2014.

[24] M. A. Acevedo, C. J. Corrada-Bravo, H. Corrada-Bravo, L. J. Villanueva-Rivera, and T. M. Aide, "Automated classification of bird and amphibian calls using machine learning: A comparison of methods," Ecological Informatics, vol. 4, no. 4, pp. 206-214, 2009.

[25] L. Pina, L. Rajamanickam, and S. Ng, "A hybrid model for pattern recognition of marine turtle species," International Journal of Scientific Engineering and Technology, vol. 5, no. 10, pp. 491-495, 2016.

[26] T. M. Cover and P. E. Hart, "Nearest neighbor pattern classification," Information Theory, IEEE Transactions on, vol. 13, no. 1, pp. 21-27, 1967.

[27] M. A. Hearst, S. T. Dumais, E. Osuna, J. Platt, and B. Scholkopf, "Support vector machines," IEEE Intelligent Systems and their applications, vol. 13, no. 4, pp. 18-28, 1998.

[28] Mergulhadores, "Tartaruga de pente," 2014. Last accessed 01 Nov 2015.

[29] G. Bradski, “Opencv library,” Dr. Dobb’s Journal of Software Tools, 2000.

[30] R. Kohavi, "A study of cross-validation and bootstrap for accuracy estimation and model selection," in 14th Int. Joint Conf. on AI, IJCAI'95, pp. 1137-1143, 1995.

[31] Y. Sasaki et al., "The truth of the f-measure," Teach Tutor mater, vol. 1, no. 5, 2007.

[32] A. G. Villa, A. Salazar, and F. Vargas, "Towards automatic wild animal monitoring: Identification of animal species in camera-trap images using very deep convolutional neural networks," Ecological Informatics, vol. 41, pp. $24-32,2017$.

\section{Authors}

Wdnei R da Paix ao has a bachelor's degree in Information System from the Federal Institute of Education, Science and Technology of Esp'irito Santo (2016), with experience abroad by the Sciences Without Borders program in Australia. Currently attending a Master's Degree in Control and Automation Engineering at the Federal Institute of Education, Science and Technology of Espirito Santo. He has experience in the area of Information Systems, with emphasis on Intelligent Computer Systems, working mainly on the following topics: information technology, computer

vision and artificial intelligence.

Thiago M. Paix ao has a bachelor's in Computer Science from the Federal University of Minas Gerais (2006), Master's degree from the University of So Paulo (2010). He works in the areas of image / video processing, computer vision (tracking and segmentation), structural pattern recognition, document reconstruction. Current occupation: Professor at the Federal Institute of Esp'irito Santo - Campus Serra (IFES) 
Mateus C. B. da Costa holds a bachelor's degree in Computer Science from the Federal University of So Carlos (1992), a Master's degree in Electrical Engineering from the State University of Campinas (1995) and a $\mathrm{PhD}$ in Computer Science from the Federal University of Minas Gerais (2008). He held a postdoctoral degree by LINA - Computer Laboratory of Nantes-Atlantique University of Nantes FRANCE from January 2011 to January 2012, in the area of Complex Event Processing. He is currently an e $\square$ ective professor at the Federal Institute of Esp'irito Santo. He has broad experience in the area of Computer Science and has more experience as a researcher in Software Engineering, Integration of Information Systems and Event Processing.

Jefferson O. Andrade received the title of Computer Engineer in 1995, and the title of Master in Computer Science in 2001, both by the Federal University of Esprito Santo. He has several years of experience as a team leader in software development projects, both in local and multinational companies in Brazil. From 2005 to 2008 he was a member of the Programming Logic Group at the University of Tsukuba, Japan. In 2013 he received his Doctorate in Education from Universidad del Norte in Paraguay (revalidated by UFPR in 2016) for his research on the application of gamification in the teaching of formal logic to undergraduate students of the Information Systems course. Currently Dr. Andrade is a full professor of the Information Technology Coordination of the Federal Institute of Education, Science and Technology of Esprito Santo. His research interests include formal methods of software development, formal systems verification, model verification, multi-valued and probabilistic logics, logical teaching, and formal methods.

Flavio G. Pereira holds a degree in Electrical Engineering (2004), a Master's degree in Electrical Engineering (Automation) (2006) and a $\mathrm{PhD}$ in Electrical Engineering (Automation) (2012) all from the Federal University of Esp'irito Santo. The subject of his dissertation was Navigation and Deviation of Obstacles Using a Mobile Robot Equipped with Laser Scanning Sensor. The theme of his thesis was People Detection and Gesture Recognition for Interaction and Cooperation between Human Beings and Robots. Performed part of the research activities of Master in the Institute of Automatic (INAUT) of the National University of San Juan (UNSJ), Argentina. During the PhD, he also carried out research at the Technische Universitt Kaiserslautern, Germany, in the area of cooperation and interaction between men and robots. He is currently Professor Federal Institute of Esp'irito Santo (IFES). Has experience in the area of Electrical Engineering, with emphasis on Mobile Robotics, Image Processing and Interaction between Men and Robots.

Karin S. Komati has been a professor at the Federal Institute of Esp'irito Santo since 2012. She holds a bachelor's degree in Computer Science from the Federal University of Esp'irito Santo (1995), and a degree in Electrical Engineering from the Federal University of Esp'irito Santo (1997). These two areas are reflected in postgraduate studies, since she holds a $\mathrm{PhD}$ in Electrical Engineering from the Federal University of Esp'irito Santo (2011) and a Master's Degree in Informatics from the Federal University of Esp'írito Santo (2002). She has been teaching higher education since 1998, working in several private and public institutions and coordinating the course of Electrical Engineering at Univix. Previously, she was systems analyst for the multinational company Xerox (1994-1998) and partner-owner of micro-enterprise services in systems development (1999-2003). In 2006, she worked in Web development at Softcreate in Japan. The research area focuses on Digital Image Processing, Pattern Recognition and Database. She is leader of the group $\mathrm{Nu}[\mathrm{Tec}]$. Currently acting as Research Coordinator for Campus Serra. She was Director of Research, Graduate and Extension for more than 3 years, responsible for the Business incubator of Campus Serra and led the proposal for a new postgraduate course "Professional Master's Degree in Control and Automation Engineering" submitted to CAPES in 2014 and approved at the 155th meeting of the CAPES CTC-ES. 
International Journal of Artificial Intelligence and Applications (IJAIA), Vol.9, No.2, March 2018

INTENTIONAL BLANK 